\title{
Characteristic flows on signed graphs and short circuit covers
}

\author{
Edita Máčajová* Martin Škoviera* \\ Department of Computer Science \\ Faculty of Mathematics, Physics and Informatics \\ Comenius University \\ 84248 Bratislava, Slovakia \\ \{macajova, skoviera\}@dcs.fmph.uniba.sk
}

Submitted: Dec 4, 2014; Accepted: Aug 3, 2016; Published: Aug 19, 2016

Mathematics Subject Classifications: 05C21, 05C22

\begin{abstract}
We generalise to signed graphs a classical result of Tutte [Canad. J. Math. 8 (1956), 13-28] stating that every integer flow can be expressed as a sum of characteristic flows of circuits. In our generalisation, the rôle of circuits is taken over by signed circuits of a signed graph which are known to occur in two types - either balanced circuits or pairs of disjoint unbalanced circuits connected with a path intersecting them only at its ends. As an application of this result we show that a signed graph $G$ admitting a nowhere-zero $k$-flow has a covering with signed circuits of total length at most $2(k-1)|E(G)|$.
\end{abstract}

Keywords: signed graph, integer flow

\section{Introduction}

It is well known that every integer flow on a graph can be expressed as a sum of characteristic flows of circuits. By a characteristic flow $\chi_{C}$ of a circuit $C$ in a graph $G$ we mean a flow that takes values +1 or -1 on $C$ and value 0 anywhere else in $G$. One way of seeing this fact is to fix an orientation of $G$, take an arbitrary spanning tree $T$ of $G$, and express the given flow $\phi$ as

$$
\phi=\sum_{x \in E(G)-E(T)} \phi(x) \cdot \chi_{T(x)}
$$

*Supported by the grants APVV-15-0220, VEGA 1/0474/15, and VEGA 1/0876/16. 
where $T(x) \subseteq T+x$ denotes the fundamental cycle corresponding to a cotree edge $x$, and the sum extends over all cotree edges of $G$. Note that the orientation of $G$ induces an orientation of each fundamental cycle $T(x)$ of $G$. It may therefore happen that an edge $t$ of $T$ belonging to two fundamental circuits $T(y)$ and $T(z)$ will receive two opposite orientations from them; equivalently, for the same direction of $t$ one can have $\chi_{T(y)}(t)=$ $-\chi_{T(z)}(t)$. It turns out, however, that unit flows of different sign through the same edge can always be avoided by a suitable choice of the set of circuits. Indeed, in 1956 Tutte [12, 6.2 ] proved that the decomposition of $\phi$ into characteristic flows can always be performed in such a way that all the circuits occurring in the expression are directed circuits with respect to a suitable fixed orientation of $G$. The aim of this paper is to establish a similar result for flows on signed graphs using the concept of a signed circuit and its characteristic flow, which we explain in Section 2 and Section 3, respectively.

Our main result reads as follows.

Theorem. For every integer flow $\phi$ on a signed graph $G$ there exists a set $\mathcal{C}$ of signed circuits of $G$ which are consistently directed with respect to a suitable orientation of $G$ and positive integers $n_{C}$ such that

$$
\phi=\sum_{C \in \mathcal{C}} n_{C} \chi_{C}
$$

This theorem is a natural generalisation of Tutte's decomposition result in the sense that its restriction to balanced signed graphs immediately yields Tutte's theorem. Rather than following Tutte's approach based on the use of chain groups we prove our theorem directly by employing a purely graph theoretical approach that inspects circuits and their signed analogues.

An important feature of our result is that, in signed graphs, a decomposition into characteristic flows may be impossible if we are restricted to integer-valued flows. Indeed, there exist fairly simple examples of signed graphs carrying integer flows that admit no decomposition into a sum of integer-valued characteristic flows. Nevertheless, as soon as we allow half-integer values, all integer flows on signed graphs become decomposable. The idea of using half-integers seems to have been independently taken up by Chen and Wang [4] where a more involved approach to the same problem is developed. (For a related work describing the structure of indecomposable integer-valued flows via their lifts to the signed double cover see [5].)

Our paper is divided into four sections. Section 2 reviews the basic concepts of signed graph theory with the emphasis on the notions related to flows. In Section 3 we introduce the concept of a characteristic flow of a signed circuit and prove our main result. In the final section we apply the main result to the study of the signed analogue of the shortest circuit cover problem recently initiated in [9].

\section{Signed graphs and flows}

A signed graph is a graph in which each edge is labelled with a sign, + or - . An orientation, or a bidirection, of a signed graph is obtained by dividing each edge into 
two half-edges and by assigning individual orientations to them subject to the following compatibility rule: a positive edge has one half-edge directed from and the other halfedge directed to its end-vertex, while a negative edge has both half-edges directed either towards or from their respective end-vertices. Thus each edge, irrespectively of its sign, has two possible orientations which are opposite to each other.

Given an abelian group $A$, an $A$-flow on a signed graph $G$ is an assignment of an orientation and a value from $A$ to each edge in such a way that for each vertex of $G$ the sum of incoming values equals the sum of outgoing values (Kirchhoff's law). If $0 \in A$ is not used as a flow value, the flow is said to be nowhere-zero. The concept of a nowherezero $A$-flow is particularly interesting when $A$ is the group of integers. A major problem is to determine, for a given signed graph $G$, the smallest integer $k \geqslant 2$ such that $G$ has an integer flow with values in the set $\{ \pm 1, \pm 2, \ldots, \pm(k-1)\}$; such a flow is called a nowhere-zero $k$-flow. In 1983, Bouchet [3] conjectured that every signed graph that admits a nowhere-zero integer flow has a nowhere-zero 6-flow. Although various approximations of this conjecture have been proved $[6,11,13,14,16]$, this conjecture remains open.

Signed graphs that admit a nowhere-zero integer flow are called flow-admissible. By contrast with unsigned graphs, flow-admissible signed graphs are not so easy to describe. For this purpose we need the notions of a balance of a signed graph and that of a signed circuit.

A circuit of a signed graph $G$ is called balanced if it contains even number of negative edges, otherwise it is called unbalanced. A signed graph itself is called balanced if it does not contain any unbalanced circuit, and is called unbalanced if it does. The collection of all balanced circuits is the most fundamental characteristic of a signed graph: signed graphs having the same underlying graphs and the same sets of balanced circuits are considered to be identical, irrespectively of their actual signatures.

A signed circuit of a signed graph is a subgraph of any of the following three types:

(1) a balanced circuit,

(2) the union of two unbalanced circuits that meet at a single vertex, or

(3) the union of two disjoint unbalanced circuits with a path connecting the circuits and meeting them only at its ends.

A signed circuit falling under item (2) or (3) is called an unbalanced bicircuit. Note that a bicircuit from item (2) can be regarded as a special case of the one from (3), with the connecting path being trivial. Observe, however, that signed circuits from items (1) or (2) admit a nowhere-zero 2-flow while those under item (3) admit a nowhere-zero 3-flow, but not a nowhere-zero 2-flow.

The following result is due to Bouchet [3, Proposition 3.1] and reflects the fact that signed circuits are inclusion minimal signed graphs that admit a nowhere-zero integer flow.

Theorem 1. A signed graph $G$ admits a nowhere-zero integer flow if and only if each edge of $G$ belongs to a signed circuit. 


\section{Decomposition into characteristic flows}

Let $\phi$ be a flow on a signed graph $G$. If we reverse the orientation of any edge $e$ and replace the value $\phi(e)$ with $-\phi(e)$, the resulting valuation will again be a flow. We regard this operation as a way of expressing the same flow $\phi$ in terms of a different orientation. In this sense, we may choose an orientation of $G$ arbitrarily as long as it complies with the signature of $G$. If $\phi$ is an integer flow, we can always find an orientation for $G$ such that $\phi(e) \geqslant 0$ for each edge $e$. We call this orientation a positive orientation of $G$ with respect to $\phi$. If $\phi$ is nowhere-zero, this orientation is unique.

Another useful operation that preserves flows on a signed graph is known as switching. It consists in choosing a vertex $v$ of $G$, reversing the orientation of each half-edge incident with $v$, and changing the signature of $G$ accordingly. As a result, all non-loop edges incident with $v$ change their signs while loops retain their signs. Since the total sign of every circuit is left unchanged, this operation gives rise to an identical signed graph equipped with a new compatible orientation. If $G$ carries a flow, then the same function works as a flow for the new signature and orientation. The same flow is thus again expressed in terms of a different orientation and signature. By a repeated use of switching we may turn a given signature into any other equivalent signature [15], keeping the flow invariant.

For a fixed orientation of $G$, the sum $\phi+\psi$ of two flows $\phi$ and $\psi$ is defined by setting $(\phi+\psi)(e)=\phi(e)+\phi(e)$; clearly, $\phi+\psi$ is again a flow. We are now interested in the reverse process of expressing an arbitrary integer flow as a sum of suitably chosen elementary flows. The question whether this is possible for every flow on an arbitrary flow-admissible signed graph was posed to us by André Raspaud (personal communication) referring to a result of Tutte $[12,6.2]$ for unsigned graphs. In Tutte's theorem, elementary flows are represented by characteristic flows of circuits. Theorem 1 suggests that in the case of flows on signed graphs circuits should be replaced with signed circuits.

Consider a pair of adjacent edges $e$ and $f$ sharing a vertex $v$ in a bidirected signed graph. We say that $e$ is consistently directed with $f$ at $v$, or that the walk $e f$ is consistently directed at $v$, if exactly one of the two half-edges incident with $v$ is directed to $v$. A path or a balanced circuit is said to be consistently directed if all pairs of consecutive edges are consistently directed. An unbalanced circuit is consistently directed if it has a single vertex, called the faulty vertex, such that all pairs of consecutive edges are consistently directed except for the pair sharing the faulty vertex. Finally, an unbalanced bicircuit is said to be consistently directed if every pair of adjacent edges in the bicircuit is consistently directed except for the two edges of either circuit that share an end-vertex of the connecting path. These vertices are the faulty vertices of the bicircuit. Examples of consistently directed signed circuits are displayed in Fig. 1.

It is easy to see that any signed circuit in a bidirected signed graph can be turned into a consistently directed signed circuit by only reversing the orientation edges. Furthermore, if the signature is switched at some vertex, consistency of the orientation is not affected. Hence, any signed circuit may have several different consistent orientations.

Definition. Let $G$ be a signed graph and let $C$ be a signed circuit of $G$ endowed with 

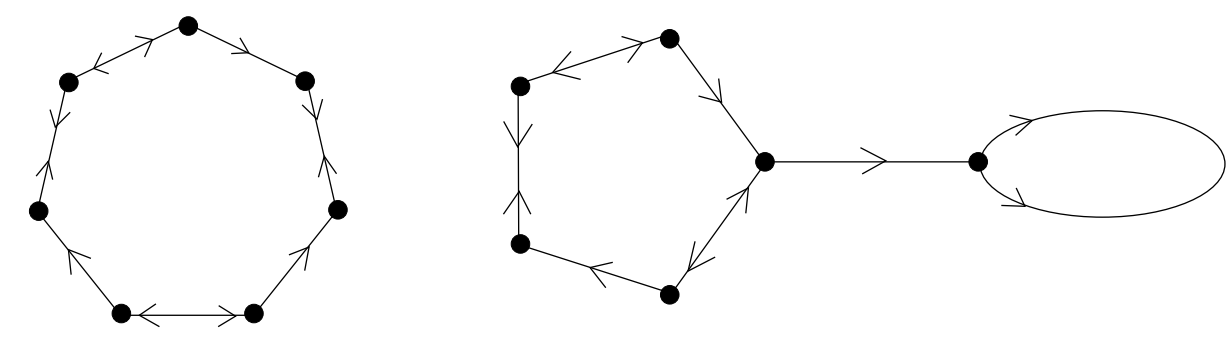

Figure 1: Consistently directed signed circuits

a consistent orientation. The characteristic flow of $C$ in $G$ is a function $\chi_{C}: E(G) \rightarrow$ $\{0,1 / 2,1\}$ defined as follows. If $C$ is a balanced circuit, we set $\chi_{C}(e)=1$ for each edge of $C$ and $\chi_{C}(e)=0$ otherwise. If $C$ is an unbalanced bicircuit, we set $\chi_{C}(e)=1$ whenever $e$ belongs to the connecting path of $C, \chi_{C}(e)=1 / 2$ whenever $e$ belongs to a circuit of $C$, and $\chi_{C}(e)=0$ otherwise. If $C$ is not consistently oriented, then its orientation can be obtained from a consistent orientation $C$ by a sequence of edge reversals or vertex switches, and the characteristic flow is defined by reversing the values of edges with reverted orientation.

Note that the characteristic flow $\chi_{C}$ of a signed circuit $C$ is a flow although not necessarily an integer flow. The values of $\chi_{C}$ and, in fact, the values of an arbitrary integral linear combination of characteristic flows of signed circuits of a graph are contained in the subgroup $(1 / 2) \mathbb{Z}$ of the additive group $\mathbb{Q}$ consisting of all integer multiples of $1 / 2$. This group, known as the group of half-integers, includes the group of integers as a subgroup of index 2. The elements of $(1 / 2) \mathbb{Z}-\mathbb{Z}$ will be called fractional. A flow with values in $(1 / 2) \mathbb{Z}$ will be called a half-integer flow.

We proceed to the main result, the decomposition theorem. For the proof recall that the support of a flow $\phi$, denoted by $\operatorname{supp}(\phi)$, is the set of all edges $e$ for which $\phi(e) \neq 0$.

Theorem 2. Let $\phi$ be an integer flow on a signed graph $G$. Then there exists a set $\mathcal{C}$ of signed circuits of $G$ which are consistently directed with respect to a positive orientation of $G$ and positive integers $n_{C}$, indexed by the elements of $\mathcal{C}$, such that

$$
\phi=\sum_{C \in \mathcal{C}} n_{C} \chi_{C}
$$

Remark. As previously indicated, fractional values in the definition of a characteristic flow are necessary for this theorem to be true. For example, let us consider the signed graph $G$ consisting of two vertices joined by a pair of positive parallel edges with a negative loop attached at either vertex. It is easy to see that $G$ admits a nowhere-zero 2-flow, but any decomposition of this flow into the sum of characteristic flows will contain characteristic flows of two distinct unbalanced bicircuits, each with coefficient 1 . The reader can easily extend this example into an infinite series of similar examples where a nowhere-zero 2-flow only decomposes into the sum of characteristic flows of two distinct unbalanced bicircuits, each with coefficient 1 . Without using fractional values these flows would be indecomposable. For a detailed study of such flows see [4]. 
Proof of Theorem 2. Throughout the proof we keep fixed a positive orientation of $G$ with respect to $\phi$. The proof is trivial if $\phi=0$, so we may assume that $\operatorname{supp}(\phi) \neq \emptyset$. If $\operatorname{supp}(\phi)$ contains a consistently directed balanced circuit $B$, we form the flow $\phi-\chi_{B}$ which is again an integer flow on $G$. We repeat the procedure with the flow $\phi-\chi_{B}$ and continue as long as the support of the current flow contains a consistently directed balanced circuit. Eventually we obtain a set $\mathcal{B}$ of consistently directed balanced circuits and the flow $\phi_{1}=\phi-\sum_{B \in \mathcal{B}} \chi_{B}$. If $\phi_{1}=0$, then $\phi=\sum_{B \in \mathcal{B}} \chi_{B}$, and the required expression for $\phi$ follows immediately. Otherwise $\operatorname{supp}\left(\phi_{1}\right)$ is nonempty and induces a subgraph $G_{1}$ which carries a non-null integer flow $\phi_{1}$. Clearly, $G_{1}$ contains no consistently directed balanced circuit.

Our next aim is to show that $G_{1}$ contains a consistently directed unbalanced bicircuit. To this end, we first identify a consistently directed unbalanced circuit in $G_{1}$. We pick an arbitrary vertex $u_{1}$ of $G_{1}$ and choose an edge $e_{1}$ that leaves $u_{1}$; since $G_{1}$ has a positive orientation such an edge always exists. Let $u_{2}$ be the other end of $e_{1}$. At $u_{2}$, there must be an edge $e_{2}$ such that the walk $e_{1} e_{2}$ is consistent at $u_{2}$. We continue in the same manner until we reach a vertex previously visited, say $u_{i}$. The segment between the two occurrences of $u_{i}$ is clearly a circuit $D$ of $G_{1}$. By the construction, $D$ is consistent everywhere except possibly $u_{i}$. Since $G_{1}$ contains no consistently directed balanced circuit, $D$ is a consistently directed unbalanced circuit and $u_{i}$ is its faulty vertex.

Next we show that $D$ is contained in a directed unbalanced bicircuit. The edges of $D$ incident with $u_{i}$ are either both directed to $u_{i}$ or both from $u_{i}$. Since $G_{1}$ has a positive orientation, there is an edge $f_{1}$ incident with $u_{i}$ which is consistent at $u_{i}$ with any of the two edges of $D$ incident with $u_{i}$. Set $v_{1}=u_{i}$ and let $v_{2}$ denote the other end of $f_{1}$. At $v_{2}$, there must be an edge $f_{2}$ such that the walk $f_{1} f_{2}$ is consistent at $v_{2}$. Again, we continue similarly until we reach a vertex $v_{j}$ that either belongs to $D$ or coincides with a vertex $v_{m}$ with $m<j$. Observe that $v_{j}$ does not lie on $D-v_{1}$, for if it does, we can divide $D$ into two $v_{j}-v_{1}$-segments $D_{1}$ and $D_{2}$ exactly one of which is consistently directed with $f_{j-1}$ at $v_{j}$. But then one of $f_{1} f_{2} \ldots f_{j-1} D_{1}$ or $f_{1} f_{2} \ldots f_{j-1} D_{2}$ is a consistently directed balanced circuit, a contradiction (see Fig. 2 for illustration). It follows that $v_{j}=v_{m}$ for some $m<j$. In this case, however, $D^{\prime}=f_{m} f_{m+1} \ldots f_{j-1}$ is a consistently directed unbalanced circuit which together with $D$ and the path $f_{1} f_{2} \ldots f_{m-1}$ forms a consistently directed unbalanced bicircuit $U$. It may happen that $u_{m}=v_{i}$ in which case the connecting path of the bicircuit is trivial.

We now take the characteristic flow $\chi_{U}$, construct the flow $\phi_{2}=\phi_{1}-\chi_{U}$, and set $G_{2}=\operatorname{supp}\left(\phi_{2}\right)$. Note that $\phi_{2}$ is not an integer flow anymore, but its fractional values are confined to two edge-disjoint consistently directed unbalanced circuits of $G_{2}$. Furthermore, $G_{2}$ contains no consistently directed balanced circuit because such a circuit would also be contained in $G_{1}$, which is impossible. The next step is to show that $G_{2}$ contains a consistently directed unbalanced bicircuit $U^{\prime}$ such that $\phi_{3}=\phi_{2}-\chi_{U^{\prime}}$ is either an integer flow or is a half-integer flow with all fractional values appearing on two edge-disjoint consistently directed unbalanced circuits. Since neither $\operatorname{supp}\left(\phi_{2}\right)$ nor $\operatorname{supp}\left(\phi_{3}\right)$ contain consistently directed balanced circuits, repeating this procedure will necessarily terminate with a set $\mathcal{U}$ of consistently directed unbalanced bicircuits such that $\phi_{1}-\sum_{U \in \mathcal{U}} \chi_{U}=0$, 


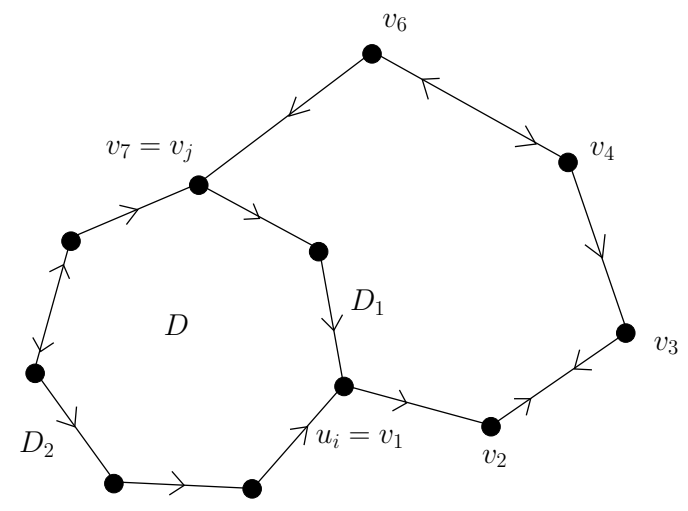

Figure 2: Contradiction in constructing an unbalanced bicircuit

implying that

$$
\phi=\sum_{B \in \mathcal{B}} \chi_{B}+\sum_{U \in \mathcal{U}} \chi_{U}
$$

Since the latter expression immediately yields the statement of the theorem, all that remains is to describe a procedure that starts with a half-integer flow $\psi$ on $G$ such that

(1) $\operatorname{supp}(\psi)$ contains no consistently directed balanced circuit, and

(2) fractional values of $\psi$ occur in exactly two edge-disjoint consistently directed unbalanced circuits of $\operatorname{supp}(\psi)$,

and constructs an unbalanced bicircuit $U^{\prime} \subseteq \operatorname{supp}(\psi)$ such that $\psi-\chi_{U^{\prime}}$ is either an integer flow or is a half-integer flow with all fractional appearing on two edge-disjoint consistently directed unbalanced circuits.

Let $\psi$ be a flow on $G$ such that $\operatorname{supp}(\psi)$ satisfies (1) and (2) stated above. Let $D$ and $D^{\prime}$ be two edge-disjoint consistently directed unbalanced circuits, and let $d$ and $d^{\prime}$ be the faulty vertices of $D$ and $D^{\prime}$, respectively. To construct an unbalanced bicircuit $U^{\prime}$ let us take the circuit $D$, choose an edge $e$ incident with $d$ which is consistently directed with either of the two adjacent edges of $D$ and proceed by successively constructing a consistently directed trail $T$ until we either reach a previously encountered vertex of $T$ or a vertex of $D \cup D^{\prime}$. Let $t$ be the terminal vertex of $T$.

First observe that $t$ cannot belong to $D-d$. Indeed, otherwise we could split $D$ into two $t$-d-segments $D_{1}$ and $D_{2}$ producing circuits $T D_{1}$ and $T D_{2}$ one of which would be a consistently directed balanced circuit in $\operatorname{supp}(\psi)$, contradicting $(2)$.

There remain four possibilities for the position of $t$ to consider.

Case 1. The vertex $t$ coincides with a previously encountered vertex of $T$, possibly $t=d$. It follows that the portion of $T$ between the two occurrences of $t$ forms a consistently directed unbalanced circuit, say $D^{\prime \prime}$, and thus $D \cup T$ forms a consistently directed unbalanced bicircuit, the sought $U^{\prime}$. Indeed, $D^{\prime}$ and $D^{\prime \prime}$ are edge-disjoint and $\left(D \cup D^{\prime}\right) \cap\left(D \cup D^{\prime \prime}\right)=D$, hence the support of the flow $\psi-\chi_{U^{\prime}}$ again contains two edge-disjoint consistently directed unbalanced circuits carrying all fractional values of $\psi-\chi_{U^{\prime}}$, namely $D^{\prime}$ and $D^{\prime \prime}$. 
Case 2. The vertex $t$ belongs to $D^{\prime}-d^{\prime}$. Let $D_{1}^{\prime}$ and $D_{2}^{\prime}$ denote the two $t$ - $d^{\prime}$-segments of $D^{\prime}$. Then exactly one of $T D_{1}^{\prime}$ and $T D_{2}^{\prime}$, say $T D_{1}^{\prime}$, is a consistently directed $d$ - $d^{\prime}$-path. Starting from $d^{\prime}$ construct a consistently directed trail $T^{\prime}$ whose first edge is consistently directed with either of the two adjacent edges of $D^{\prime}$ and continue until we either reach a previously visited vertex of $T^{\prime}$ or a vertex of $D \cup D^{\prime} \cup T$. Let $t^{\prime}$ be the first such vertex.

Observe that $t^{\prime}$ belongs neither to $D-d$ not to $D^{\prime}-d^{\prime}$. Otherwise, in the former case $D$ would contain a $t^{\prime}$-d-segment $S$ such that $S T D_{1}^{\prime} T^{\prime}$ is a consistently directed balanced circuit in $\operatorname{supp}(\psi)$, and similarly in the latter case $D^{\prime}$ would contain a $t^{\prime}$ - $d^{\prime}$-segment $S^{\prime}$ such that $S^{\prime} T^{\prime}$ is a a consistently directed balanced circuit in $\operatorname{supp}(\psi)$. In both cases we would get a contradiction.

There remain two possibilities for the position of $t^{\prime}$.

Subcase 2.1. The vertex $t^{\prime}$ coincides with a previously encountered vertex of $T^{\prime}$, possibly $t^{\prime}=d^{\prime}$. The portion of $T^{\prime}$ between the two occurrences of $t^{\prime}$ forms a consistently directed unbalanced circuit, say $D^{\prime \prime}$, and hence $D^{\prime} \cup T^{\prime}$ is a consistently directed unbalanced bicircuit. If we set $U^{\prime}=D^{\prime} \cup T^{\prime}$, then the support of the flow $\psi-\chi_{U^{\prime}}$ again contains two edge-disjoint directed unbalanced circuits carrying all fractional values of $\psi-\chi_{U^{\prime}}$, namely $D$ and $D^{\prime \prime}$.

Subcase 2.2. The vertex $t^{\prime}$ belongs to $T$, possibly $t^{\prime}=d$. It is obvious that $t^{\prime} \neq t$ because otherwise $t^{\prime}$ would lie in $D^{\prime}-d^{\prime}$, which we have shown to be impossible. On the other hand, $t^{\prime}$ may coincide with $d$. The vertex $t^{\prime}$ splits the path $T$ into two segments, a $d$ - $t^{\prime}$-segment $W_{1}$, which may be trivial, and a $t^{\prime}$-t-segment $W_{2}$, which is nontrivial. Consider the last edge $g$ of $T^{\prime}$ and the first edge $h$ of the segment $W_{2}$. If $g$ was consistently directed with $h$ at $t^{\prime}$, then $W_{2} D_{1}^{\prime} T^{\prime}$ would be a consistently directed balanced circuit within $\operatorname{supp}(\psi)$, which is impossible. Thus $g$ is not consistently directed with $h$ at $t^{\prime}$. Since $h$ is consistently directed at $t^{\prime}$ with the last edge of $W_{1}$, provided that $t^{\prime} \neq d$, or with both edges of $D$ incident with $d$, provided that $t^{\prime}=d$, it follows that $W_{1} \cup T^{\prime}$ is a consistently directed path whose ends $d$ and $d^{\prime}$ are the only faulty vertices of $D \cup W_{1} \cup T^{\prime} \cup D^{\prime}$. Thus $D \cup W_{1} \cup T^{\prime} \cup D^{\prime}$ is a consistently directed unbalanced bicircuit, the sought $U^{\prime}$. It is now easy to see that $\psi-\chi_{U^{\prime}}$ is an integer flow whose support does not contain any consistently balanced circuit.

Case 3. The vertex $t$ coincides with $d^{\prime}$ and the terminal edge of $T$ is consistently directed at $d^{\prime}$ with either of the two adjacent edges of $D^{\prime}$. In this case $D \cup T \cup D^{\prime}$ is a consistently directed unbalanced bicircuit. We can set $U^{\prime}=D \cup T \cup D^{\prime}$ and observe that $\psi-\chi_{U^{\prime}}$ is an integer flow whose support contains no consistently directed balanced circuit. Again, the required conclusion holds.

Case 4. The vertex $t$ coincides with $d^{\prime}$ but the terminal edge of $T$ is not consistently directed at $d^{\prime}$ with the two adjacent edges of $D^{\prime}$. Since $G$ has a positive orientation, there exists an edge in $\operatorname{supp}(\psi)$ incident with $d^{\prime}$ which is consistently directed at $d^{\prime}$ with both adjacent edges of $D^{\prime}$. Hence, starting from this edge we can again construct a consistently directed trail $T^{\prime}$ which terminates by reaching either a previously visited vertex of $T^{\prime}$ or a vertex of $D \cup D^{\prime} \cup T$. As in Case 2, the terminal vertex $t^{\prime}$ cannot lie in $(D-d) \cup\left(D^{\prime}-d^{\prime}\right)$ for otherwise we could find a consistently directed balanced circuit in 
$\operatorname{supp}(\psi)$, contradicting $(2)$. There remain two possibilities for the position of $t^{\prime}$ which are completely analogous to Subcases 2.1 and 2.2, and are therefore left to the reader.

As we have seen, in each case the procedure can either be continued or will terminate with the zero flow. The proof is complete.

Observe that the proof of Theorem 2 makes no use of the characterisation of flowadmissible graphs given in Theorem 1. Just on the contrary, Theorem 1 easily follows from Theorem 2 .

Corollary 3. A signed graph $G$ admits a nowhere-zero integer flow if and only if each edge of $G$ belongs to a balanced circuit or an unbalanced bicircuit.

Proof. The forward implication is an immediate consequence of Theorem 2. For the converse, let $\mathcal{C}=\left\{C_{1}, C_{2}, \ldots, C_{r}\right\}$ be a set of signed circuits such that each edge of $G$ belongs to a member of $\mathcal{C}$. We first fix an arbitrary orientation of $G$; note that the elements of $\mathcal{C}$ need not be consistently directed with respect to this orientation. Now we can define the function $\psi: E(G) \rightarrow \mathbb{Z}$ by setting

$$
\psi=\sum_{i=1}^{r} 2^{2 i-1} \chi_{C_{i}} .
$$

It is easy to see that $\psi$ is indeed a nowhere-zero integer flow on $G$.

\section{Application to signed circuit covers}

A circuit cover of an unsigned graph is a collection of circuits such that each edge of the graph belongs to at least one of the circuits. It is a standard problem to find, for a given graph, a circuit cover of minimum total length. It has been conjectured that every bridgeless graph $G$ has a circuit cover of length at most $7|E(G)| / 5$ (Jaeger, private communication; independently [1]), but the best current general bound is $5|E(G)| / 3$ (see $[1,2])$. The $7 / 5$-conjecture is particularly interesting for its relationship to other prominent conjectures in graph theory. For instance, its validity is implied by the Petersen flow conjecture (alternatively known as the Petersen colouring conjecture) of Jaeger [7, Section 7], while the conjecture itself implies the celebrated cycle double cover conjecture (see Raspaud [10] and Jamshy and Tarsi [8]).

A natural analogue of a circuit cover for signed graphs is the concept of a signed circuit cover introduced in [9]. It is a collection $\mathcal{C}$ of signed circuits of a signed graph $G$ such that each edge of $G$ is contained in at least one member of $\mathcal{C}$. In [9] it was shown that every flow-admissible signed graph $G$ has a signed circuit cover of total length at most $11|E(G)|$

We now apply our Theorem 2 to the shortest signed circuit problem. If a signed graph $G$ admits a nowhere-zero integer $k$-flow $\phi$, we can decompose it into the sum $\sum_{C \in \mathcal{C}} n_{C} \chi_{C}$ of characteristic flows guaranteed by Theorem 2. Obviously, the set $\mathcal{C}$ provides a signed circuit cover of $G$. This cover yields the following bounds. 
Corollary 4. If a signed graph $G$ has a nowhere-zero flow $\phi$, then $G$ has a signed circuit cover such that each edge e belongs to at most $2|\phi(e)|$ signed circuits.

Corollary 5. If a signed graph $G$ admits a nowhere-zero $k$-flow, then it has a signed circuit cover of total length at most $2(k-1)|E(G)|$.

Observe that if Bouchet's 6-flow conjecture [3] is true, then the previous corollary implies that every flow-admissible signed graph $G$ has a signed circuit cover of total length at most $10|E(G)|$.

\section{References}

[1] N. Alon and M. Tarsi. Covering multigraphs by simple circuits. SIAM J. Algebraic Discrete Methods, 6:345-350, 1985.

[2] J.-C. Bermond, B. Jackson, and F. Jaeger. Shortest covering of graphs with cycles. J. Combin. Theory Ser. B, 35:297-308, 1983.

[3] A. Bouchet. Nowhere-zero integral flows on a bidirected graph. J. Combin. Theory Ser. B, 34:279-292, 1983.

[4] B. Chen and J. Wang. Classification of integral flows on signed graphs. arXiv:1112.0642v2 (June 2013).

[5] B. Chen, J. Wang, and T. Zaslavsky. Resolution of irreducible integral flows on a signed graph. manuscript.

[6] M. DeVos. Flows on bidirected graphs. arXiv:1310.8406 (October 2013).

[7] F. Jaeger. Nowhere-zero flow problems. In Selected Topics in Graph Theory Vol. 3 (L. W. Beineke, R. J. Wilson, Eds.), pages 71-95. Academic Press, London, 1988.

[8] U. Jamshy and M. Tarsi. Short cycle covers and the cycle double cover conjecture. J. Combin. Theory Ser. B, 56:197-204, 1992.

[9] E. Máčajová, A. Raspaud, E. Rollová, and M. Škoviera. Circuit covers of signed graphs. J. Graph Theory, 81(2):120-133, 2016.

[10] A. Raspaud. Flots et couvertures par des cycles dans les graphes et les matroïdes, Thèse de $3^{\text {ème }}$ cycle, Université de Grenoble, 1985.

[11] A. Raspaud and X. Zhu. Circular flow on signed graphs. J. Combin. Theory Ser. B., 101: 464-479, 2011.

[12] W. T. Tutte. A class of Abelian groups. Canad. J. Math., 8:13-28, 1956.

[13] E. Wei, W. Tang, and X. Wang. Flows in 3-edge-connected bidirected graphs. Front. Math. China, 6:339-348, 2011.

[14] R. Xu and C.-Q. Zhang. On flows in bidirected graphs. Discrete Math., 299:335-343, 2005.

[15] T. Zaslavsky. Signed graphs. Discrete Appl. Math., 4:47-74, 1982; Erratum, ibid. 5:248, 1983.

[16] O. Zýka. Nowhere-zero 30-flows on bidirected graphs. KAM Series No. 87-26, Charles University, Prague, 1987. 